Research Article

\title{
Electrical Resistivity of Pristine and Functional Single-Wall Carbon Nanotubes
}

\author{
Yijiang Lu, ${ }^{1}$ Jing Li, ${ }^{1}$ and Haiping Hong ${ }^{2}$ \\ ${ }^{1}$ Nanotechnology Branch, NASA Ames Research Center, Moffett Field, Sunnyvale, CA 94035, USA \\ ${ }^{2}$ Department of Material and Metallurgical, South Dakota School of Mines and Technology, Rapid City, SD 57701, USA \\ Correspondence should be addressed to Haiping Hong; haiping.hong@sdsmt.edu
}

Received 6 January 2013; Accepted 16 February 2013

Academic Editor: Yun Zhao

Copyright (c) 2013 Yijiang Lu et al. This is an open access article distributed under the Creative Commons Attribution License, which permits unrestricted use, distribution, and reproduction in any medium, provided the original work is properly cited.

\begin{abstract}
The resistance of several pristine and functional single-wall carbon nanotubes (SWNTs) deposited and dried on interdigitated electrode (IDE) chips was investigated to better understand how functional groups influence their resistivity. Without the external electrical field, the resistance was generally increased for the sulfonated and fluorinated SWNTs but not for COOH-SWNTs. With a $3 \mathrm{~V}$ electric field applied during depositing, while no change in resistance was found for the purified pristine SWNTs, fluorinated SWNTs, COOH SWNTs, and Ni-SWNTs, a significant decrease in resistance was observed in sulfonated SWNTs and unpurified pristine SWNTs, which could be due to the alignment of SWNTs in an electric field. The alignment of the sulfonated SWNTs is most likely due to the charge of the sulfate functional group. It is interesting to note that the alignment was found in the unpurified pristine SWNTs but not in the purified pristine ones which have lessened resistivity. The lower resistivity in the purified pristine SWNTs may be due to the smaller number $(<5 \%)$ of impurities. The significance of this research is that hydrophilic COOH-SWNTs could be a better candidate than the hydrophobic pristine SWNTs for being used in many applications, especially in polymer nanocomposites.
\end{abstract}

\section{Introduction}

Carbon nanotubes have attracted extensive attention recently because of their extraordinary thermal, electrical, and mechanical properties [1-3]. Due to these unique properties, they have the potential to be used in electrical devices [4], nanofluids [5, 6], grease [7], and sensors [8-10].

Measuring the electrical properties of carbon nanotubes in solutions is not easy because it is difficult to get a homogeneous solution with carbon nanotubes. It is very hard to dissolve or disperse the carbon nanotubes in their pristine form in the hydrophilic fluids, such as water and ethylene glycol, simply because carbon nanotubes are inorganic solids (hydrophobic) [11]. Usually chemical surfactants are added to disperse the nanotubes. But the added chemical surfactant is always a concern in such measurements because it would complicate the interpretation of electrical conductivity results.

An alternative approach to improve the solubility of carbon nanotubes in water or organic solvents is by surface modification, such as covalent, noncovalent, free-radical, and electrochemical modification or plasma treatments [12]. The most used and efficient method is chemical functionalization, through which carboxyl groups or other functional groups such as fluorinated groups and sulfonated groups are attached to the sidewall or the ends of the single-walled carbon nanotubes (SWNTs), leading to a reduction of the strong van der Waals interactions among carbon nanotubes and strongly facilitating the separation of SWNT bundles into individual tubes [12]. For example, recently, Dr. Billups' group at Rice University has synthesized highly exfoliated, water soluble, and single-walled carbon nanotubes with functional aryl sulfonated group [13]. Therefore, no chemical surfactant is necessary to disperse the nanotubes, thus eliminating the influence of chemical surfactants and simplifying the experimental procedure. The addition of the functional groups to the SWNTs causes a change of the electronic structure from a trigonal-planar local bonding geometry to a tetrahedral geometry, leading to the transformation of $\mathrm{sp}^{2}$ - into $\mathrm{sp}^{3}$ hybridized carbon atoms [12], which may affect the intrinsic 
electronic properties of SWNTs and their potential application in electronic devices and sensors. Therefore, it is interesting to investigate the effect of the added functional groups on electrical resistance to better understand how the chemical bonds and geometric/electronic configuration affect the electrical properties of the SWNTs.

In this paper, the electrical resistance of several pristine and functionalized SWNTs was investigated with and without an electric field to better understand the relationship between nanotube structure and physical properties (thermal, electrical, and mechanical).

\section{Experimental and Procedure}

The functional sulfonated SWNTs were obtained from Dr. Billups' group, Rice University. The details for the synthesis of these materials can be found in [13]. The COOH SWNTs were obtained from School of Mines and Nankai University respectively. (fluorinated single-wall carbon nanotubes) FSWNTs were purchased from Carbon Nanotechnologies Incorporation (CNI, Houston, TX, USA). The surfaces have not been treated chemically or physically. Ni-coated SWNTs were prepared using the methodology as reported previously $[14,15]$. A handheld sonic probe (Sonic Dismembrator, Model 100) was used to efficiently disperse the nanotubes into solvents. All flocculation of the nanotubes visible to the eye was removed by this process.

The nanotube coatings on the interdigitated electrode (IDE) chips were applied by a Nanoject II (Drummond Scientific Co.). The smallest drop distributed by this device is $2.3 \mathrm{~nL}$. Ten drops of each sample were put to each IDE chip to ensure a uniform $23 \mathrm{~nL}$ SWNTs coating. When an electrical field was applied, $3 \mathrm{~V}$ or $5 \mathrm{~V}$ was added to the IDE chips before dropping the solution droplets on the electrodes. At the same time, the IDE sensor chip is heated up to $60^{\circ} \mathrm{C}$. The electric field is removed once the SWNTs are dried. The IDE is made by a P-type boron-doped silicon (100) wafer with a resistivity of $0.006-0.01 \mathrm{ohm}-\mathrm{cm}$ and thickness of $500 \pm 25 \mu \mathrm{m}$. A layer of $0.5 \mu \mathrm{m}$ silicon dioxide was thermally grown on top of the Si substrates. A layer of $200 \mathrm{~nm}$ platinum on top of $20 \mathrm{~nm}$ titanium was deposited on the $\mathrm{SiO}_{2}$ layer with the designed finger patterns. These patterns consisted of $4,8,12$, and $50 \mu \mathrm{m}$ finger gaps with $10 \mu \mathrm{m}$ finger width [16]. The IDE used for the conductivity measurement is shown in Figure 1 with a finger width of $10 \mu \mathrm{m}$ and a gap width of $12 \mu \mathrm{m}$.

The electrical conductivities of various fillers (nanotubes) in solid phase were measured by Keithley 2002/7001 High performance DMM/Multipluxer and Agilent 34401 DMM (precisely calibrated). A Keithley 237 High voltage measure unit was used to measure the voltage of the electric field when the nanotube coating was applied.

\section{Results and Discussion}

In order to better understand the influence of functional groups on the conductivity (resistivity) of nanotubes, the electrical resistivity of various fillers (nanotubes) in solid phase was measured. Table 1 lists seven nanofillers (nanotubes) with different functional groups and chemical structures. All solutions were prepared to make SWNTs in the similar

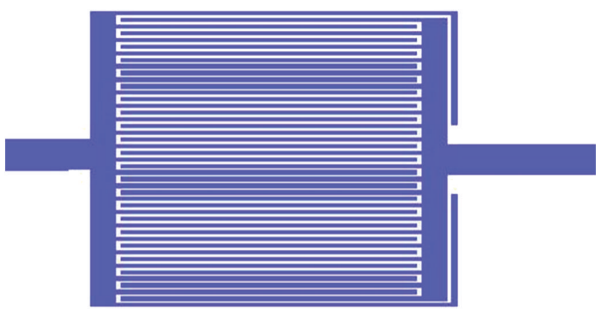

FIGURE 1: Interdigitated electrode (IDE) chip for measuring the resistance of different SWNTs.

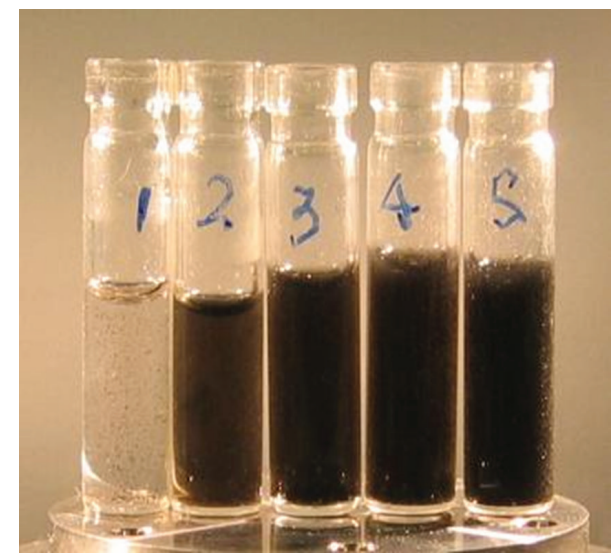

Figure 2: Picture of five nanofiller (nanotube) solutions with different functional groups and chemical structures. (1) Unpurified pristine SWNT, (2) fluorinated SWNT, (3) COOH SWNT (school of mines), (4) COOH SWNT (outside campus), and (5) sulfonated SWNT.

TABLE 1: Types of nanofillers, concentrations, and solvents.

\begin{tabular}{lccc}
\hline No. & Type of nanofiller & Concentration, wt $\%$ & $\begin{array}{c}\text { Solvent } \\
\text { type }\end{array}$ \\
\hline 1 & Unpurified pristine SWNT & $0.0324 \%$ & DMF \\
2 & Fluorinated SWNT & $0.0364 \%$ & DMF \\
3 & $\begin{array}{c}\text { COOH SWNT } \\
\text { (school of mines) }\end{array}$ & $0.0363 \%$ & DMF \\
4 & COOH SWNT & $0.0365 \%$ & DMF \\
5 & (outside campus) & $0.0366 \%$ & DMF \\
6 & Sulfonated SWNT & $0.037 \%$ & DMF \\
7 & Purified pristine SWNT & $0.037 \%$ & DMF \\
\hline
\end{tabular}

concentration by $\mathrm{wt} \%$ in order to control the amount of nanotubes deposited on each IDE.

Pristine SWNTs were not distributed as well as the others in Dimethylformamide (DMF) even after being set in an ultrasonicator for 15 minutes, see Figure 2. The possible reason is that the pristine nanotube is hydrophobic. The other functional SWNTs dispersed better in DMF because they are hydrophilic.

Table 2 lists the conductivity (resistivity) data of the SWNTs with and without an applied electric field when depositing the nanotubes onto the electrodes. 
TABLE 2: Resistance of SWNTs with and without an applied electric field.

\begin{tabular}{|c|c|c|c|}
\hline Coating sample & $\begin{array}{c}\text { Agilent } \\
\text { 34401 DMM }\end{array}$ & $\begin{array}{c}\text { Keithley } \\
2002 \text { DMM }\end{array}$ & Comments \\
\hline $\begin{array}{l}\text { Unpurified pristine } \\
\text { SWNT }\end{array}$ & $256 \mathrm{~K} \Omega$ & $270 \mathrm{~K} \Omega$ & \\
\hline $\begin{array}{l}\text { Unpurified pristine } \\
\text { SWNT* }^{*}\end{array}$ & $29.9 \Omega$ & $29.9 \Omega$ & $\begin{array}{l}\text { Applied } \\
3 \mathrm{~V}\end{array}$ \\
\hline Fluorinated SWNT & $41.2 \mathrm{~K} \Omega$ & $40.5 \mathrm{~K} \Omega$ & \\
\hline Fluorinated SWNT* & $39.1 \mathrm{~K} \Omega$ & $39.2 \mathrm{~K} \Omega$ & $\begin{array}{l}\text { Applied } \\
3 \mathrm{~V}\end{array}$ \\
\hline COOH SWNT(S3) & $12.0 \Omega$ & $12.0 \Omega$ & \\
\hline COOH SWNT(S3)* & $16.1 \Omega$ & $16.2 \Omega$ & $\begin{array}{l}\text { Applied } \\
3 \mathrm{~V}\end{array}$ \\
\hline COOH SWNT(S4) & $18.5 \Omega$ & $18.2 \Omega$ & \\
\hline $\mathrm{COOH}$ SWNT(S4)* & $17.8 \Omega$ & $17.8 \Omega$ & $\begin{array}{c}\text { Applied } \\
3 \mathrm{~V}\end{array}$ \\
\hline Sulfonated SWNT & $5.34 \mathrm{~K} \Omega$ & $5.29 \mathrm{~K} \Omega$ & \\
\hline Sulfonated SWNT* & $25.6 \Omega$ & $25.7 \Omega$ & $\begin{array}{l}\text { Applied } \\
3 \mathrm{~V}\end{array}$ \\
\hline Ni-SWNT & $17.6 \Omega$ & & \\
\hline Ni-SWNT* & $26.5 \Omega$ & & $\begin{array}{c}\text { Applied } \\
3 \mathrm{~V}\end{array}$ \\
\hline Purified pristine SWNT & $21.5 \Omega$ & & \\
\hline Purified pristine SWNT* & $34.1 \Omega$ & & $\begin{array}{c}\text { Applied } \\
3 \mathrm{~V}\end{array}$ \\
\hline
\end{tabular}

* An electric field of $3 \mathrm{~V}$ across the fingers with a gap size of $12 \mathrm{~mm}$ was applied while the SWNT samples were being coated on the IDE. Instrument used for the resistance measurement: Keithley 2002 digital multimeter and Agilent 34401 DMM.

When no electric field is applied, the resistance of the sulfonated SWNTs and fluorinated SWNTs is $5.34 \mathrm{k} \Omega$ and $41.5 \mathrm{k} \Omega$, respectively, which are about two to three orders of magnitude higher than that of purified pristine ones with a value of $21.5 \Omega$, indicating that functionalization generally increases the resistance except for $\mathrm{COOH}-\mathrm{SWNT}$ and NiSWNTs, with a resistance range from $12.0 \Omega$ to $17.8 \Omega$, close to or even lower than $21.5 \Omega$ of purified pristine ones. It is reported that coating nickel on the SWNT aids in exfoliation of SWNTs agglomerates and enables good dispersions [17]. The enhanced dispersions and formation of continuous interconnections between SWNTs lead to the low electrical resistance of Ni-SWNTs. In addition, coating the SWNTs with nickel will not diminish the sidewall properties and affect the electrical properties as in sulfonated SWNTs and fluorinated SWNTs [17], resulting in a much lower electric resistance in Ni-SWNTs than that of other functional SWNTs.

When a $3 \mathrm{~V}$ electric field was applied during coating the SWNTs onto IDE chips, the resistance decreased from $5.34 \mathrm{k} \Omega$ to $25.6 \Omega$ for sulfonated SWNTs and from $256 \mathrm{k} \Omega$ to $29.9 \Omega$ for unpurified pristine SWNTs, respectively. The decrease in resistance is most likely caused by the nanotubes alignment. In our previous study $[15,18]$, a significantly increase in thermal conductivity was observed in the nanofluids containing magnetic-metal-coated carbon nanotubes when a magnetic field was applied. Real images showed that the initial randomly dispersed Ni-coated SWNTs gradually stretched and finally aligned to form chains and clusters which are longer than the real length of individual nanotubes [18]. The contact between the individual nanotubes in these chains and clusters leads to the increase in thermal conductivity of the nanofluids. Similarly, since the sulfated SWNTs have charged sulfate functional groups, these charges could make SWNTs align in an electric field, leading to the contact enhancements among the nanotubes, thus decreasing the electrical resistance. No change in resistance was found in the purified pristine SWNTs when they were applied in an electric field, which indicates that there is no alignment for the purified pristine SWNTs in an electric field because there is no charged functional group. The same thing (no alignment) should also be expected for the unpurified pristine SWNTs because they are the same except that there are more impurities (e.g., metal catalysts and their oxides and graphite) in the unpurified ones. Therefore, these impurities in the unpurified pristine SWNTs could be the reason for the unexpected alignment.

The resistance of the Ni-SWNTs does not change in a $3 \mathrm{~V}$ electric field but change in a $5 \mathrm{~V}$ electric field (data is not listed) because Ni-SWNTs are slightly polarized in nature due to the addition of Ni. These slightly polarized Ni-SWNTs will align along the electric field when the field is strong enough ( $5 \mathrm{~V}$ in this study) and leads to the decrease in electric resistance. It was reported that $\mathrm{Cu}$ and $\mathrm{Ti}$ atoms adsorbed on SWNTs are positively charged and the two nearest carbon atoms carry a little negative charge based on the model using pseudo-potential plane wave method [19]. Such charge transfer increased several times when an electric field was applied. The binding energy of Ni to SWNT is very close to that of $\mathrm{Ti}$ but larger than that of $\mathrm{Cu}$ [20]. Therefore, similar charge transfer would be expected in Ni-SWNTs, and it would increase with the strength of an electric field, leading to the polarization of the Ni-SWNTs and their alignment.

No significant change was observed for the resistance of the fluorinated SWNTs and COOH SWNTs after an electric field was applied to the IDE during SWNTs deposition. This is due to that the $\mathrm{COOH}$ and fluorine functional groups have no charges, and, therefore, they were not aligned under an electric field. It is also worth noting that the resistance of $\mathrm{COOH}$ SWNTs is three magnitudes lower than that of fluorinated SWNTs. The much lower resistance of $\mathrm{COOH}$ SWNTs is because that, during the well-controlled carboxyl functinalization, the chemical modification is limited mostly to the opening of the tube caps at the ends and the formation of functional groups at defect sites along the sidewall. SWNTs functionalized in this manner basically retain their pristine electronic properties [12]. Therefore, the resistance of the $\mathrm{COOH}-\mathrm{SWNT}$ is very similar to that of the purified pristine ones. It was found using the density functional theory that vacancy- $\mathrm{COOH}$ pairs with a strong covalent bond are energetically favorable on the zigzag nanotube sidewall after acid treatment, and the electrical conductivity would be enhanced [21]. This is the case in the present study as seen from Table 2, the resistance of the COOH-SWNTs is pretty close to and even lower than that of the pristine ones with an applied electric field or not. By comparison, addition 
reactions enable the direct coupling of functional groups such as fluorine onto the $\pi$-conjugated carbon framework of the tubes. It is known that the formation of carbon-heteroatom bonds will transform a $\mathrm{sp}^{2}$-hybridized configuration to that of a sp ${ }^{3}$-hybridized structure, leading to the disruption of the pseudo-one-dimensional lattice of the SWNTs and increase in resistance [12]. This is consistent with the results in the present study that the resistance of the fluorinated SWNTs is three magnitudes higher than that of the pristine SWNTs and COOH-SWNTs.

Previous study showed that the tensile strength of the cross-link membrane with $5 \mathrm{wt} \%$ of sulfonated SWNTs and sulfonated polystyrene is only $35 \mathrm{MPa}$, which is much lower than the expected value by considering the extremely high tensile strength of SWNTs (around $60 \mathrm{GPa}$ ) [22]. Assuming that nanotubes disperse very well in the polymer matrix, ideally, a $5 \mathrm{wt} \%$ nanotube loading in a film would have a tensile strength of around $\approx 3 \mathrm{GPa}$. One possible explanation of the lower than expected values is that the inserted functional groups interfere with the conjugate structure of the nanotube, thereby reducing its macromechanical strength. The effective electrical conductivity measurement of sulfonated SWNTs solution [11] provides strong evidence and support to this. No percolation threshold phenomenon is observed in the experiments. The linear conductivity increase versus the weight percentage indicates that the dominant conduction mechanism might be the ionic conduction, which is in agreement with the chemical structure of sulfonated SWNTs (anion $\mathrm{SO}_{3}{ }^{-}$group).

The existence of anion $\mathrm{SO}_{3}{ }^{-}$group in sulfonated SWNTs is also proved in the present study. The resistivity of the SWNTs is $5.34 \mathrm{k} \Omega$, which are about two orders of magnitude higher than that of purified pristine ones with a value of $21.5 \Omega$. It decreased from $5.34 \mathrm{k} \Omega$ to $25.6 \Omega$ under the external electrical field, probably caused by the nanotubes alignment due to the existence of the anion $\mathrm{SO}_{3}{ }^{-}$groups.

The motivation of the resistivity measurement of pristine and functionalized SWNTs is to better understand the relationship between nanotube structure and physical properties (thermal, electrical, and mechanical). Our study indicated that macrophysical strength is damaged by addition (insertion) of functional group [22]. Electrical conductivity measurement could provide additional information of structure of SWNTs. COOH-SWNTs have a resistance around $12.0 \Omega$, even lower than that of purified pristine ones with a value of $21.5 \Omega$, indicating that $\mathrm{COOH}$ group would maximally reduce the interfacial influence and keep the unbroken nanotube conjugate structure.

Changing the SWNTs from hydrophobic to hydrophilic with $\mathrm{COOH}$ group, while keeping its excellent electrical conductivity, makes it a better choice than pristine one to be used as nanofillers for various applications, especially in polymer composites [23].

\section{Conclusions}

The electrical resistivity of several pristine and functional SWNTs in solid phase was investigated with and without an applied electric field.
Without an applied electric field, the resistance generally increased for the sulfonated and fluorinated SWNTs but not for $\mathrm{COOH}-\mathrm{SWNTs}$. With an applied electric field, no change was observed in resistance for purified pristine SWNTs, COOH-SWNTs, and fluorinated SWNTs. A significant decrease in resistance was observed from sulfonated SWNTs and unpurified pristine SWNTs when an electric field was applied, indicating that they both align within an electric field. The alignment of the sulfonated SWNTs is most likely due to the charged sulfonate groups. Alignment was found in the unpurified pristine SWNT but not the purified pristine SWNTs, which indicates that the alignment of unpurified pristine SWNTs is probably due to the impurities within its structure. The lower resistivity in the purified pristine SWNTs may be due to the smaller number $(<5 \%)$ of impurities.

The results in this study show that the resistance of pristine and functional singlewall nanotubes is correlated to the type of functional groups and the alignment of the nanotubes. The significance of this research is that the hydrophilic $\mathrm{COOH}-\mathrm{SWNTs}$ show relative low resistivity, which indicates that conjugated $\pi$ structure of nanotubes (sidewall properties) is alive. These results demonstrate that hydrophilic $\mathrm{COOH}-\mathrm{SWNTs}$ could be a better candidate than the hydrophobic pristine SWNTs for being used in many applications, especially in polymer nanocomposites.

\section{Acknowledgments}

H. Hong would like to thank NASA EPSCoR (award no. NNX09AU83A) for financial support. Jing Li would like to thank for DHS HSARPAR (IAA no. HSHQDC-08-x-00870) for financial support. Thanks are due to Mianliang Huang for his useful comments and corrections.

\section{References}

[1] S. Berber, Y. K. Kwon, and D. Tomanek, "Unusually high thermal conductivity of carbon nanotubes," Physical Review Letters, vol. 84, no. 20, pp. 4613-4616, 2000.

[2] C. Zhou, J. Kong, and H. Dai, "Intrinsic electrical properties of individual single-walled carbon nanotubes with small band gaps," Physical Review Letters, vol. 84, no. 24, pp. 5604-5607, 2000.

[3] M. S. Dresselhaus, G. Dresselhaus, J. C. Charlier, and E. Hernández, "Electronic, thermal and mechanical properties of carbon nanotubes," Philosophical Transactions of the Royal Society A, vol. 362, no. 1823, pp. 2065-2098, 2004.

[4] A. Javey, R. Tu, D. B. Farmer, J. Guo, R. G. Gordon, and H. Dai, "High performance n-type carbon nanotube field-effect transistors with chemically doped contacts," Nano Letters, vol. 5, no. 2, pp. 345-348, 2005.

[5] H. Hong, X. Luan, M. Horton, C. Li, and G. P. Peterson, "Alignment of carbon nanotubes comprising magnetically sensitive metal oxides in heat transfer nanofluids," Thermochimica Acta, vol. 525, no. 1-2, pp. 87-92, 2011.

[6] J. Wensel, B. Wright, D. Thomas et al., "Enhanced thermal conductivity by aggregation in heat transfer nanofluids containing metal oxide nanoparticles and carbon nanotubes," Applied Physics Letters, vol. 92, no. 2, Article ID 023110, 3 pages, 2008.

[7] H. Hong, D. Thomas, A. Waynick, W. Yu, P. Smith, and W. Roy, "Carbon nanotube grease with enhanced thermal and electrical 
conductivities," Journal of Nanoparticle Research, vol. 12, no. 2, pp. 529-535, 2010.

[8] Y. Lu, J. Li, J. Han et al., "Room temperature methane detection using palladium loaded single-walled carbon nanotube sensors," Chemical Physics Letters, vol. 391, no. 4-6, pp. 344-348, 2004.

[9] J. Li, Y. Lu, Q. Ye, M. Cinke, J. Han, and M. Meyyappan, “Carbon nanotube sensors for gas and organic vapor detection," Nano Letters, vol. 3, no. 7, pp. 929-933, 2003.

[10] J. Li and Y. Lu, "Carbon nanotube based chemical sensors for space and terrestrial applications," ECS Transactions, vol. 19, no. 6, pp. 7-15, 2009.

[11] B. Glover, K. W. Whites, H. Hong, A. Mukherjee, and W. E. Billups, "Effective electrical conductivity of functional singlewall carbon nanotubes in aqueous fluids," Synthetic Metals, vol. 158, no. 12, pp. 506-508, 2008.

[12] K. Balasubramanian and M. Burghard, "Chemically functionalized carbon nanotubes," Small, vol. 1, no. 2, pp. 180-192, 2005.

[13] F. Liang, J. M. Beach, P. K. Rai et al., "Highly exfoliated watersoluble single-walled carbon nanotubes," Chemistry of Materials, vol. 18, no. 6, pp. 1520-1524, 2006.

[14] Y. Zhang, N. W. Franklin, R. J. Chen, and H. Dai, "Metal coating on suspended carbon nanotubes and its implication to metaltube interaction," Chemical Physics Letters, vol. 331, no. 1, pp. 3541, 2000.

[15] B. Wright, D. Thomas, H. Hong et al., "Magnetic field enhanced thermal conductivity in heat transfer nanofluids containing $\mathrm{Ni}$ coated single wall carbon nanotubes," Applied Physics Letters, vol. 91, no. 17, Article ID 173116, 3 pages, 2007.

[16] J. Li, Y. Lu, Q. Ye, L. Delzeit, and M. Meyyappan, "A gas sensor array using carbon nanotubes and microfabrication technology," Electrochemical and Solid-State Letters, vol. 8, no. 11, pp. H100-H102, 2005.

[17] D. K. Chakravarthi, V. N. Khabashesku, R. Vaidyanathan et al., "Carbon fiber-bismaleimide composites filled with nickelcoated single-walled carbon nanotubes for lightning-strike protection," Advanced Functional Materials, vol. 21, no. 13, pp. 2527-2533, 2011.

[18] M. Horton, H. Hong, C. Li, B. Shi, G. P. Peterson, and S. Jin, "Magnetic alignment of Ni-coated single wall carbon nanotubes in heat transfer nanofluids," Journal of Applied Physics, vol. 107, no. 10, Article ID 104320, 4 pages, 2010.

[19] L. Wang and Y. Zhang, "Electronic and magnetic properties of metal atom adsorption on SWNT," Physica E: Low-Dimensional Systems and Nanostructures, vol. 43, no. 4, pp. 889-892, 2011.

[20] E. Durgun, S. Dag, V. M. K. Bagci, O. Gülseren, T. Yildirim, and S. Ciraci, "Systematic study of adsorption of single atoms on a carbon nanotube," Physical Review B, vol. 67, no. 20, Article ID 201401, 4 pages, 2003.

[21] C. Wang, G. Zhou, J. Wu, B. L. Gu, and W. Duan, "Effects of vacancy-carboxyl pair functionalization on electronic properties of carbon nanotubes," Applied Physics Letters, vol. 89, no. 17, Article ID 173130, 3 pages, 2006.

[22] Y. Dai, H. Hong, M. Guiver, and J. S. Welsh, "Reinforced films based on cross-linked water-soluble Sulfonated carbon nanotubes with Sulfonated Polystyrene," Journal of Nanoscience and Nanotechnology, vol. 9, no. 9, pp. 5150-5156, 2009.

[23] Z. Han and A. Fina, "Thermal conductivity of carbon nanotubes and their polymer nanocomposites: a review," Progress in Polymer Science, vol. 36, no. 7, pp. 914-944, 2011. 

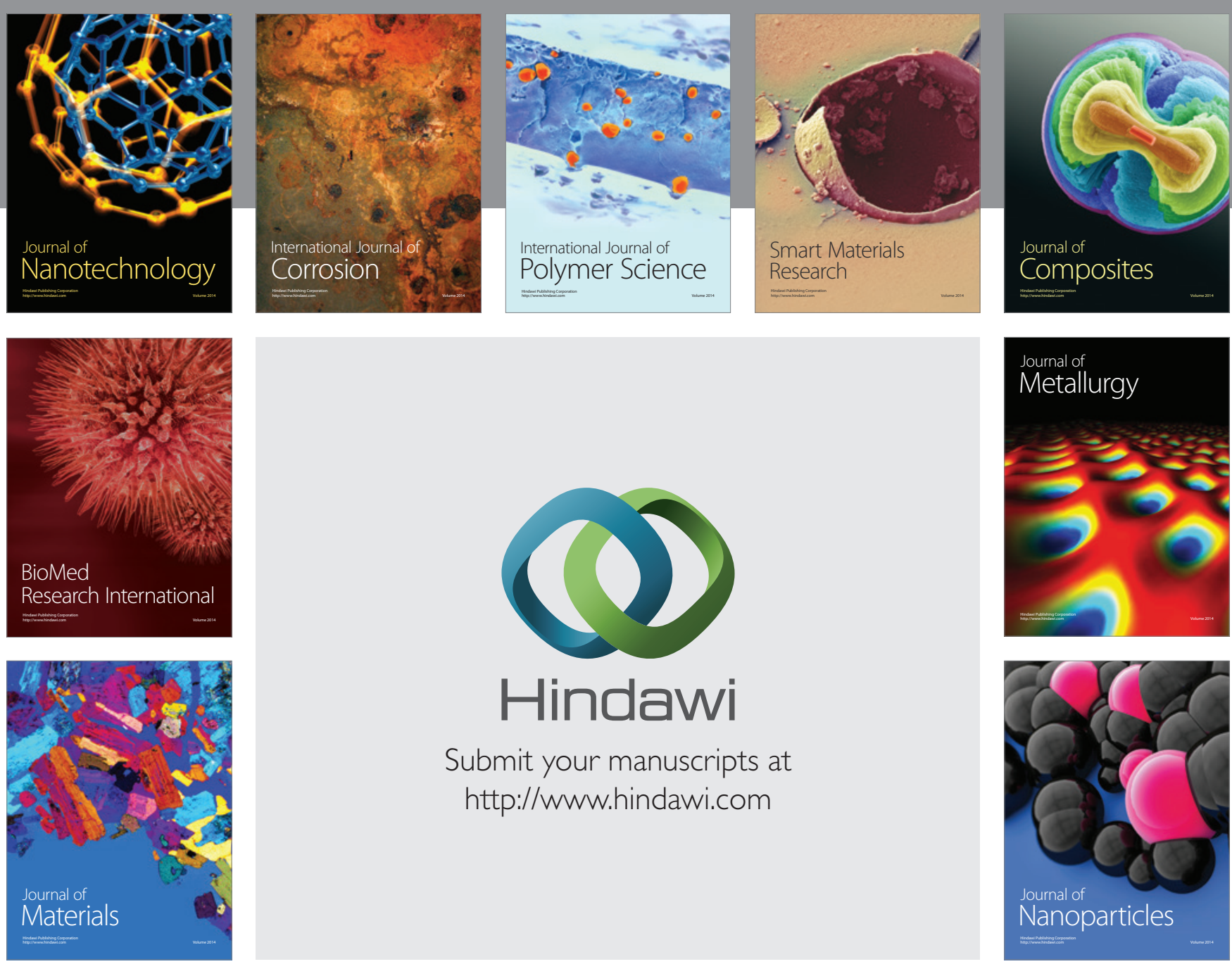

Submit your manuscripts at http://www.hindawi.com
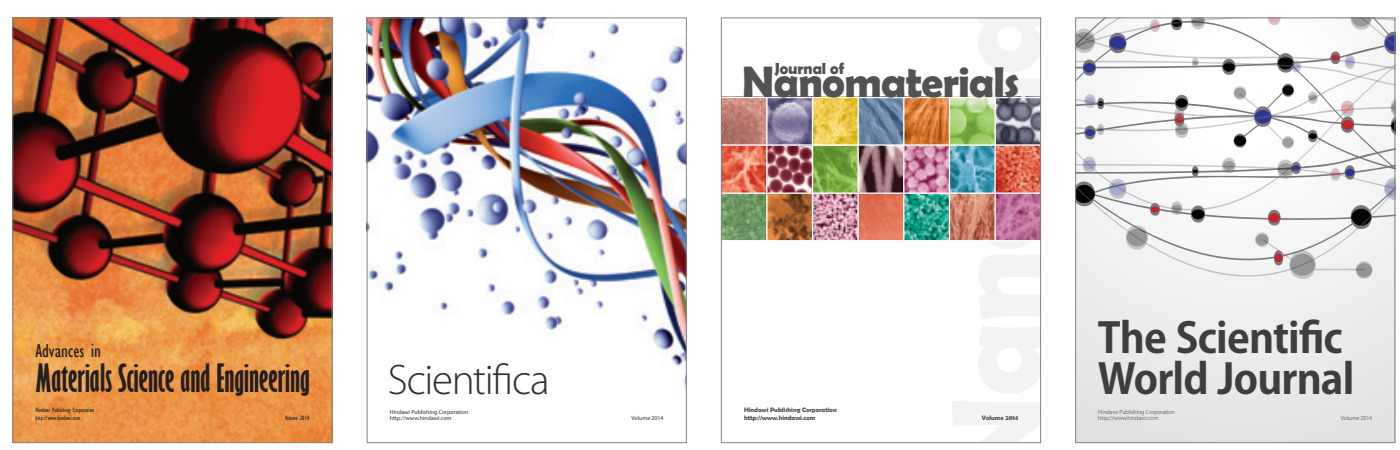

\section{The Scientific World Journal}
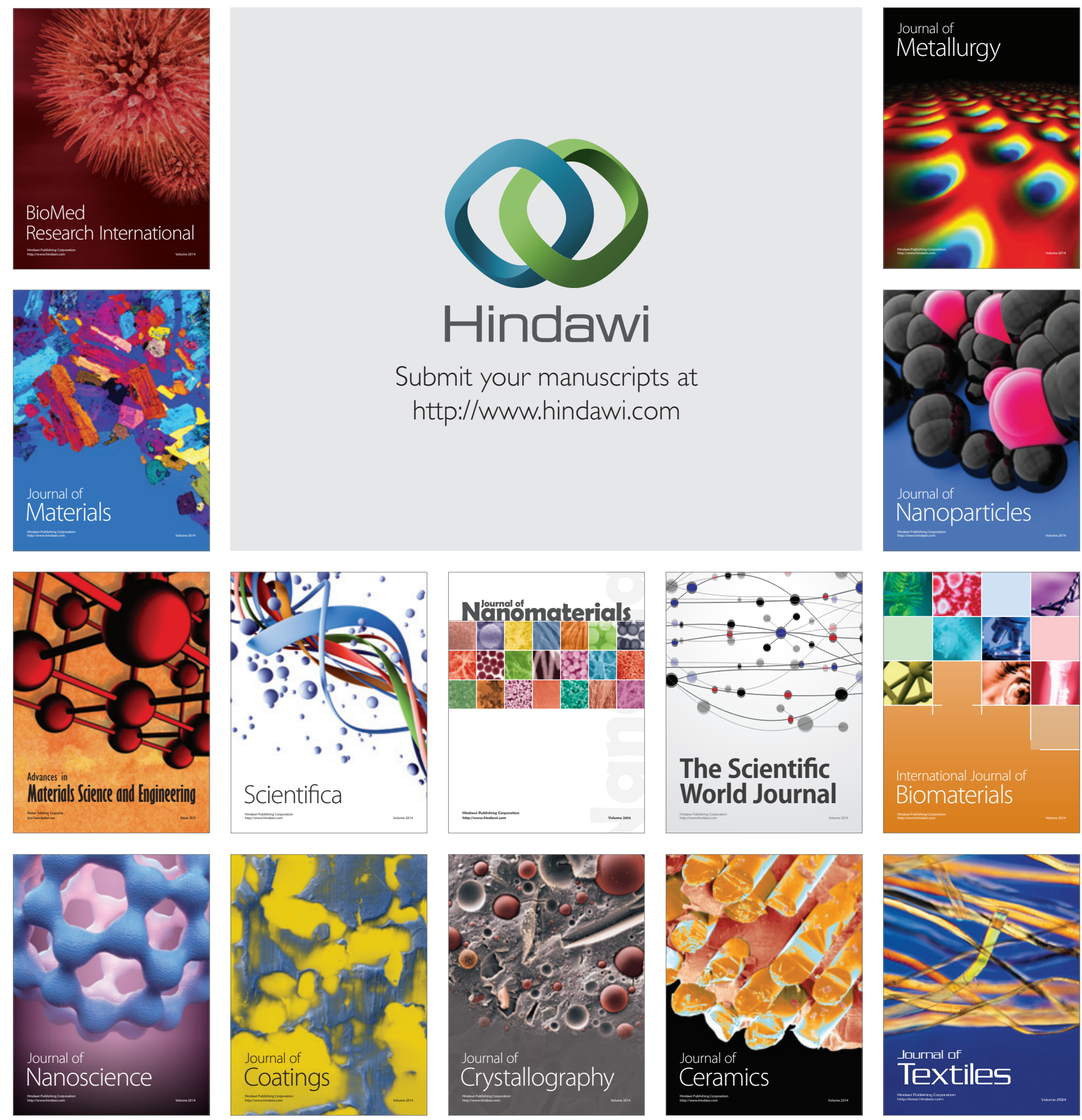